\title{
Diversity Analysis of Chinese Tibetan Naqu Yak (Bos grunniens) Populations Using mtDNA
}

\author{
Wang-Dui Basang', Tian-Wu An², Luo-Bu Danjiü, Yan-Bin Zhu' ${ }^{1}$, Shi-Cheng $\mathrm{He}^{3}$, \\ Xiao-Lin Luo ${ }^{2}$, Wei-Wei Ni ${ }^{4}$, Xiao Wang ${ }^{4}$, Shu-Zhu Cheng ${ }^{4}$, Jian Wang ${ }^{4}$ and \\ Guang-Xin E $\mathbf{E}^{4, *}$ \\ ${ }^{1}$ Institute of Animal Husbandry and Veterinary Medicine, Tibet Academy of Agriculture \\ and Animal Husandry Science, Lasa 850009, China \\ ${ }^{2}$ Sichuan Academy of Grassland Sciences, Chengdu, Sichuan 611731, China \\ ${ }^{3}$ Nagqu Grassland Station, Naqu 852000, China \\ ${ }^{4}$ College of Animal Science and Technology, Southwest University, Chongqing 400715, \\ China
}

Wang-Dui Basang and Tian-Wu An have contributed equally to this article.

\begin{abstract}
A B S T R A C T
The yak (Bos grunniens) is an indigenous domestic animal living at high altitudes in the Tibetan plateau that is economically important for the Tibetan people. In this study, we investigated the diversity and phylogeography of four geographic ecotype populations of the Naqu yak (133 individuals) using an 811-bp mitochondrial DNA D-loop region sequence. In total, 57 polymorphic sites, including 54 singlenucleotide polymorphisms and 3 single-nucleotide copy number variants, and 59 haplotypes were detected. The number of haplotypes within the population ranged from 17 (SN and JL) to 28 (DX). The haplotype diversity ranged from 0.9420 (SN) to 0.9770 (NR). The highest nucleotide diversity was found in the JL population (0.01479), whereas the lowest was found in the SN (0.00894) population. Phylogenetic analysis revealed that these four populations separated into two haplogroups; the first included SN, DX, and NR, and the second included JL. However, no significant divergence was found among the ecotype populations using a pair-wise difference comparison $\left(F_{S T}\right)$. Thus, the Naqu yak has multiple maternal origins and demonstrates high diversity within 4 geographic ecotype yak populations. In addition, the yaks exhibit some diversity based on the number of unique haplotypes within each population; however, they have not heavily and significantly diverged because all the populations share the most high-frequency haplotypes. Therefore, this study not only shows that the different ecotype populations of the Naqu yak carry high genetic diversity but also indicates that frequent genetic material exchanges have led to smaller differences in genetic divergence between different ecotypes.
\end{abstract}
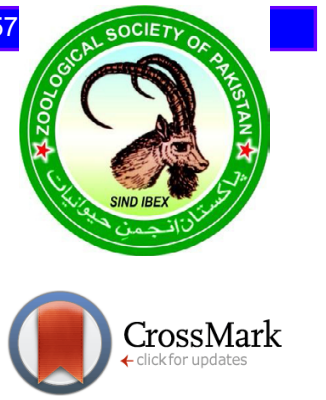

\begin{tabular}{l} 
Article Information \\
Received 29 December 2017 \\
Revised 22 February 2018 \\
Accepted 24 March 2018 \\
Available online 31 August 2018 \\
Authors' Contributions \\
WDB, TWA and GXE designed \\
the experiments and wrote the \\
manuscript. YBZ and XLL performed \\
the lab experiment and analysed the \\
data. LBD, WWN, XW, JW, SZC and \\
SCH collected the samples. \\
Key words \\
\hline Yak, Mitochondrial DNA, D-Loop \\
region, Haplotype.
\end{tabular}

\section{INTRODUCTION}

$\mathrm{T}$ he domestic yak (Bos grunniens), which was domesticated from the wild yak (Bos mutus), is a longhaired, domesticated bovid that is found throughout the Himalayan region of the Indian subcontinent, the Tibetan Plateau and as far north as Mongolia and Russia. The yak plays important food, economic, and cultural roles in the Asian range and is an important domestic animal for the local people.

Mitochondrial genome sequence (mtDNA) analysis is a popular tool to estimate the phylogenic evolution and migration of humans (e.g., Schaan et al., 2017; Hernández et al., 2017), wild (e.g., Xie et al., 2017; Ming et al., 2017) and domestic animals (Zhang et al., 2016; Kim et al., 2016; Eusebi et al., 2017). In particular, previous studies

\footnotetext{
*orresponding author: eguangxin@126.com 0030-9923/2018/0006-2051 \$ 9.00/0

Copyright 2018 Zoological Society of Pakistan
}

have investigated yak diversity and population structures in different habitat locations in China, including Gansu (Cheng et al., 2014) and Qing-Hai (e.g., Qian et al., 2013), Xin-Jiang (e.g., Wang et al., 2013) and Tibet (e.g., Zhang et al., 2012; Song et al., 2014), using mtDNA.

Naqu, which is located in the northern part of the Tibet Autonomous Region in the hinterland of the Qinghai-Tibet Plateau, is one of the main farm sources of yaks in Tibet. This region has an average altitude of more than 4500 meters.

Studies of the domestication history of the Naqu yak and estimations of the gene flow among different ecotype yak populations in this area are important and will help improve the genetics of the local yak.

\section{MATERIALS AND METHODS}

Venous blood samples were obtained from 133 individual yaks from 4 ecotype populations in the Tibetan $\mathrm{Naqu}$; their geographic information is presented in Table 
I. The blood samples were collected in EDTA tubes and frozen at $-20^{\circ} \mathrm{C}$ prior to extraction. Genomic DNA was isolated using standard procedures (Sambrook and Russell, 2001); the DNA quality was verified on a $1 \%$ agarose gel and quantified using a DTX microplate reader (Beckman Coulter, USA).

The high-variability region of the mitochondrial DNA control region (D-loop) was amplified using the mDNA-F (5'- GTA AAG AGC CTC ACC AGT AT -3') and mDNA-R (5'- GTC GGG AGA CTC ATC TAG GC - 3') from Mipam et al. (2012). PCR amplification was conducted in a PTC-100 ${ }^{\mathrm{TM}}$ PCR instrument (MJ Research, Inc., MA, USA) with a total reaction volume of $50 \mu \mathrm{L}$ containing $150 \mathrm{ng}$ of DNA, $5 \mu \mathrm{L}$ of $10 \times$ PCR standard reaction buffer, $4 \mu \mathrm{L}(10 \mathrm{pmol} / \mu \mathrm{L})$ of dNTPs, $2 \mu \mathrm{L}(50$ $\mathrm{mmol} / \mu \mathrm{L})$ of $\mathrm{MgCl}_{2}, 1 \mu \mathrm{L}(10 \mathrm{pmol} / \mu \mathrm{L})$ of each forward and reverse primer, and $2.5 \mathrm{U}$ of Taq DNA polymerase from Promega (Beijing, China). The PCR program was described in Mipam et al. (2012). The PCR products were directly sequenced using mDNA-F with the Genetic Analyzer 3130 xl (Applied Biosystems, USA).

The D-loop sequence alignments were constructed using the ClustalX software v2.0 (Larkin et al., 2007). DnaSP 5.10 (Rozas and Rozas, 1995) was used to screen haplotypes and to estimate polymorphisms and the average numbers of nucleotide differences between populations (Kxy). The best fitting model of DNA substitution for BI was obtained using jModelTest (0.1.1) (Posada, 2008). The maximum likelihood phylogenetic network of D-loops among all individuals was constructed with the MEGA
(5.0) software (Tamura et al., 2011), and the bootstrap values to support the nodes of the tree were based on 1000 iterations of the heuristic search. Pairwise differences in populations $\left(F_{S T}\right.$; Slatkin, 1995) were displayed using the Arlequin software version 3.5.1.3 (Excoffier et al., 2010). In addition, a visual haplotype phylogenetic network and frequency distribution were conducted and subjected to median-joining network analysis according to the methods of Bandelt et al. (1999) and Lyimo et al. (2014) using Network 4.1 (http://www.fluxus-engineering.com/ sharenet.htm).

\section{RESULTS}

All of the Naqu yak sequences were aligned to the complete yak mitochondrial genome (GenBank No. KM223416) (Guangxin et al., 2016). A total of 133 sequences covering bp 1 to 359 and 15872 to 16319 (811 bp total) were obtained as reference sequences (M223416) of the yak mitochondrial D-loop region and submitted to GenBank (MG213580 to MG213712). In addition, 57 polymorphic sites, including 54 single-nucleotide polymorphisms and 3 single-nucleotide copy number variants, were identified in the D-loops of these 133 individuals.

The nucleotide polymorphisms ranged from 0.00894 (SN) to $0.01479(\mathrm{JL})$. Tajima's D ranged from -1.31787 (SN) to $0.26375(\mathrm{NR})$, and the $P$-values of Tajima's D in all the populations were not significant based on the chisquare test $(P>0.10$; Table II).

Table I.- Geographic information for the sampling locations of the four Naqu yak populations.

\begin{tabular}{lcccccl}
\hline Ecotype population & Code & Sample size & Altitude (m) & East longitude & North latitude & Location \\
\hline Jili Strain & JL & 26 & 4501 & 30.64081 & 93.23253 & Jiali town, Naqu, Tibet, China \\
Neirong Strain & NR & 30 & 4619 & 32.10777 & 92.30334 & Neirong town, Naqu, Tibet, China \\
Nima Strain & SN & 24 & 4541 & 31.78470 & 87.23677 & Nima town, Naqu, Tibet, China \\
DangXiong Strain & DX & 53 & 4293 & 30.47312 & 91.10116 & Dangxiong town, Naqu, Tibet, China \\
\hline
\end{tabular}

Table II.- Nucleotide and haplotype polymorphisms of the mtDNA D-loop within the Naqu yak populations.

\begin{tabular}{|c|c|c|c|c|c|c|}
\hline \multirow[t]{2}{*}{ Population } & \multirow{2}{*}{$\begin{array}{l}\text { Sample } \\
\text { size }\end{array}$} & \multicolumn{3}{|c|}{ Nucleotide polymorphism } & \multicolumn{2}{|c|}{ Haplotype polymorphism } \\
\hline & & $\begin{array}{c}\text { Nucleotide } \\
\text { diversity }(\pi)\end{array}$ & Tajima's D & $\begin{array}{c}\text { Tajima's D } \\
\text { P-value }\end{array}$ & $\begin{array}{l}\text { Number of } \\
\text { haplotype }\end{array}$ & $\begin{array}{c}\text { Haplotype } \\
\text { diversity }\end{array}$ \\
\hline $\mathrm{JL}$ & 26 & 0.01479 & 0.13439 & $\mathrm{P}>0.1$ & 17 & 0.9600 \\
\hline NR & 30 & 0.01408 & 0.26375 & $P>0.1$ & 22 & 0.9770 \\
\hline $\mathrm{SN}$ & 24 & 0.00894 & -1.31787 & $\mathrm{P}>0.1$ & 17 & 0.9420 \\
\hline DX & 53 & 0.01196 & -0.36650 & $\mathrm{P}>0.1$ & 28 & 0.9427 \\
\hline
\end{tabular}




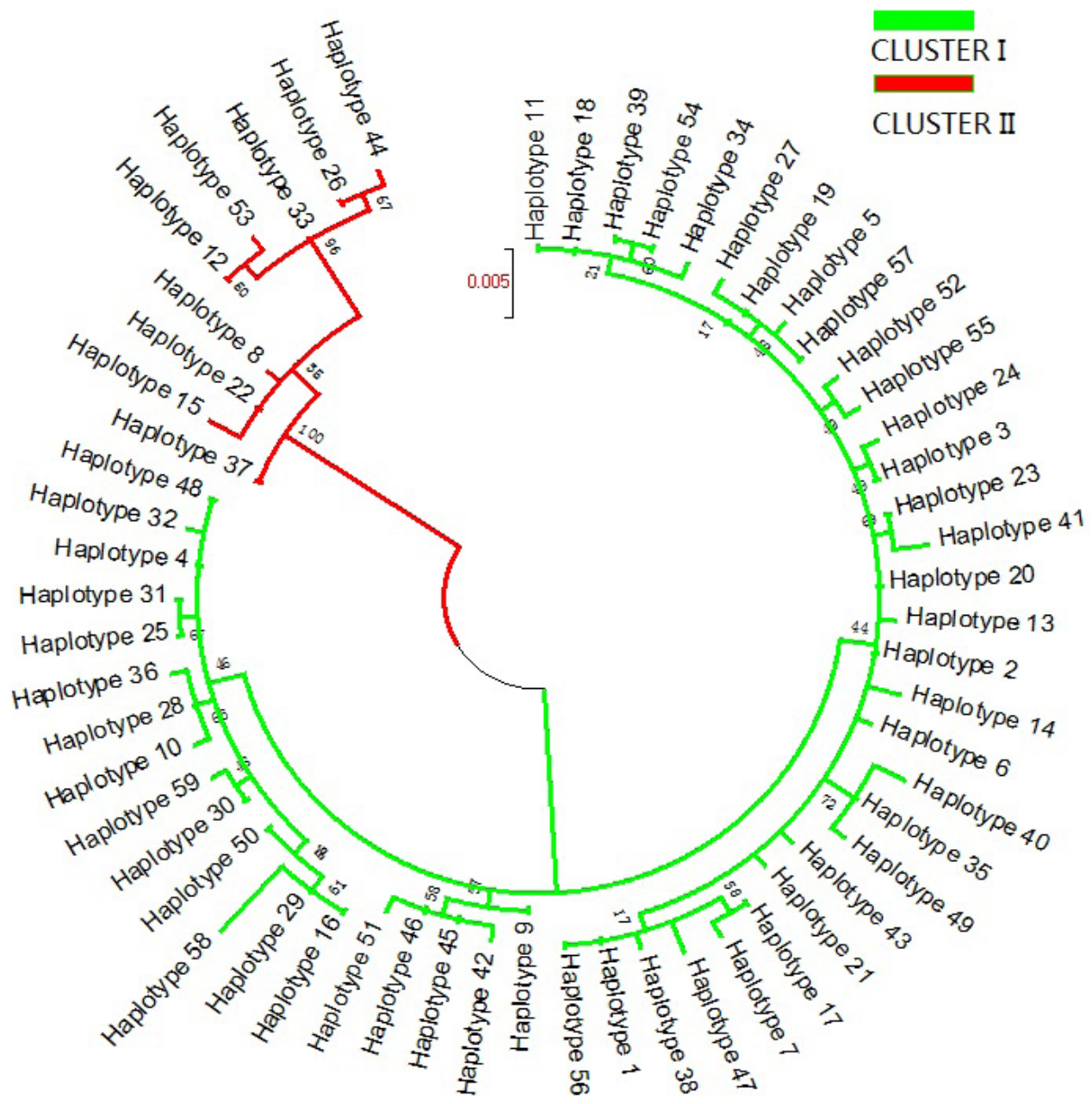

Fig. 1. Molecular phylogenetic analysis of 59 Naqu yak mtDNA D-loop haplotypes using the maximum likelihood method. The evolutionary history was inferred using the maximum likelihood method based on the Tamura-Nei model (Tamura and Nei, 1993). The tree with the highest log likelihood (-1693.9317) is shown. The percentage of trees in which the associated taxa clustered together is shown next to the branches. Initial tree(s) for the heuristic search were obtained automatically by applying the neighbor-joining and BioNJ algorithms to a matrix of pairwise distances estimated using the maximum composite likelihood (MCL) approach and then selecting the topology with a superior log likelihood value. The rate variation model allowed some sites to be evolutionarily invariable ([+I], 46.4858\% sites). The tree is drawn to scale, with branch lengths measured in the number of substitutions per site. The analysis involved 59 nucleotide sequences. A total of 811 positions were included in the final dataset.

A total of fifty-nine haplotypes were identified in the 133 individuals. The DX population carried the largest number of haplotypes (28), whereas the SN and JL populations had the smallest number of haplotypes (17). The haplotype diversity of the four populations ranged from 0.9420 (SN) to 0.9770 (JL; Table II). The phylogenetic relationship of the 59 haplotypes was constructed using the maximum likelihood method, and two haplogroups were identified from the 59 haplotypes (Fig. 1). The highest frequency haplotypes were Hapotype_2, Haplotype_4, and Haplotype_ 8, which were shared by the four populations. Additionally, 13 of the 59 haplotypes were shared by two or three populations, and 43 haplotypes were unique (Fig. 2).

Table III.- Genetic divergence between populations with $K x y$ and matrix of the pairwise $F_{S T}$

\begin{tabular}{lcccc}
\hline Code & JL & NR & SN & DX \\
\hline JL & $\backslash$ & 12.31282 & 10.46635 & 11.70972 \\
NR & -0.01721 & $\backslash$ & 10.37778 & 11.61887 \\
SN & 0.01724 & 0.00840 & $\backslash$ & 9.40330 \\
DX & 0.00452 & -0.00318 & -0.00492 & $\backslash$ \\
\hline
\end{tabular}

Above diagonal, Kxy; below diagonal, $F_{S T}$ 

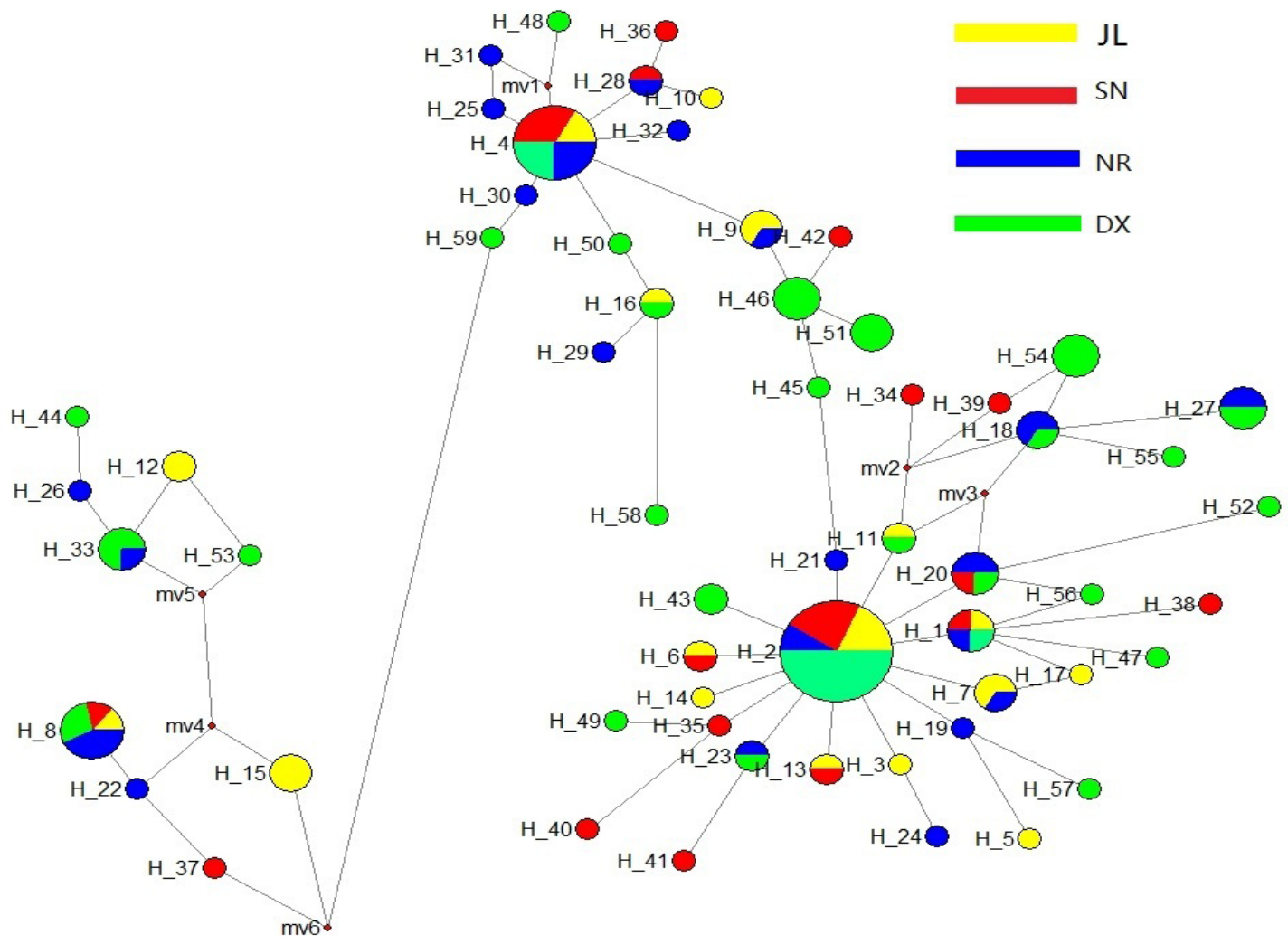

Fig. 2. Network and frequency profiles of the 59 Naqu yak haplotypes with mtDNA D-loop sequences.

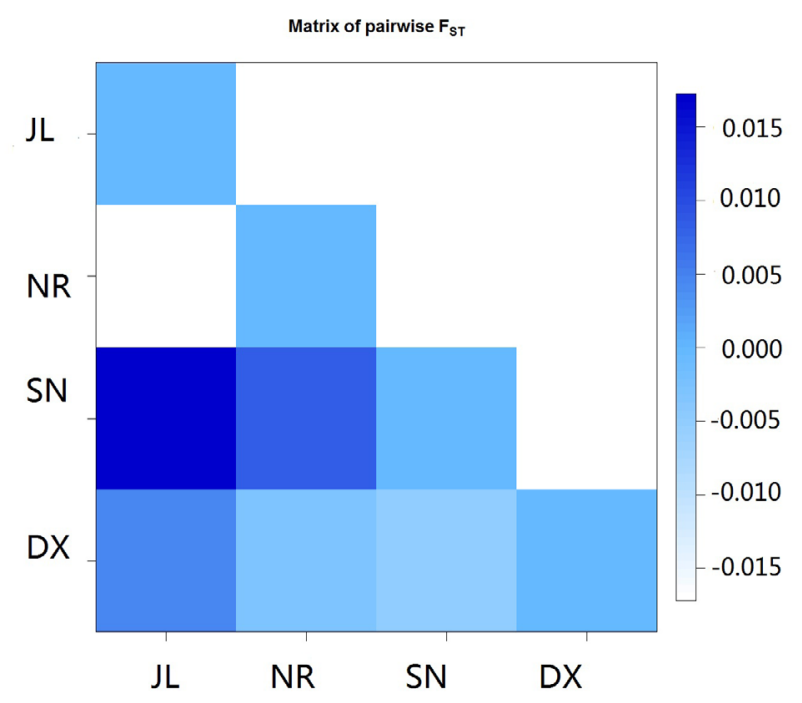

Fig. 3. Matrix of pairwise FST values of the four Naqu ecotype yak populations.

In the $F_{S T}$ analysis of the Naqu yak populations, the largest difference was found between JL and SN $\left(F_{S T}=0.01724, \quad P=0.25225 \pm 0.0445\right)$, and the smallest difference was found between JL and NR $\left(F_{S T}=-0.01721\right.$,
$P=0.71171 \pm 0.0497$ (Table III; Fig. 3). The $F_{S T}$ distribution indicated that these four populations were separated into two groups; the first group contained JL, and the second group included SN, DX, and NR, which was consistent with the phylogenetic patterns of these four populations constructed using Kxy (Fig. 4; Table III). However, no significant divergence was observed between the populations according to the chi-square test of $F_{S T}$.

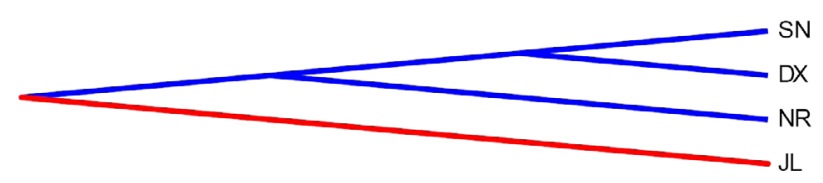

Fig. 4. Average number of nucleotide differences between populations (Kxy) among the four Naqu ecotype Yak populations based on DnaSP 5.10.

\section{DISCUSSION}

Recently, mitochondrial DNA polymorphisms have been widely used to estimate the gene flow and phylogenetic relationships of maternal lineages in domestic animals (e.g., Nguluma et al., 2017; Jia et al., 
2017; Deng et al., 2017; Almarzook et al., 2017) and wild animals (e.g., Elsner et al., 2017; Khaire et al., 2017). The domestic yak (Bos grunniens) is a large and commercially important animal living in the Qinghai-Tibetan Plateau of China with a high-altitude climate (Guangxin et al., 2016). Various research efforts have addressed the diversity and population structure analysis of domestic yaks from different geographic distributions (e.g., Guo et al., 2006; Lai et al., 2007; Mipam et al., 2012; Huang et al., 2012).

Naqu is an important habitat of the Tibetan domestic yak in the northern part of the Tibetan Autonomous Region, as well as a junction between several Chinese yak breeding areas. Therefore, studying the diversity of different Naqu yak populations and assessing the gene flow with other yak populations from neighboring areas will contribute to our understanding of their genetic diversity status and help inform conservation policies.

First, the nucleotide polymorphisms of the D-loop region in this study ranged from 0.00894 to 0.01479 ; this range was larger than the number of polymorphisms detected in the Zhongdian yak (0.00534) (Tu et al., 2016) but similar to the findings for 8 other Chinese Tibetan yak populations (0.00451 to 0.01438) (Song et al., 2014). Additionally, this value was much larger than the number of polymorphisms found in the mtDNA ND6 gene (Hai et al., 2014). This observation is consistent with the hypothesis that the D-loop region contains the most polymorphisms in the mitochondrial DNA (Tsai and St John, 2016; Gao et al., 2017). Second, in comparison with previous studies, the haplotype diversity $(\mathrm{Hi})$ of the 4 Naqu yak populations ( 0.9420 to 0.9770$)$ was higher than the diversities among the eight different known Tibetan populations (0.827 to 0.927; Song et al., 2014), the Tianzhu white yak (Gansu, China), and the Jiulong yak (Sichuan, China) but was lower than the diversity of the Maiwa yak (Sichuan, China; Lai et al., 2005). Additionally, the haplotype numbers of the four ecotype populations ranged from 17 to 28 , revealing that the Naqu yak populations carried wide and abundant genetic diversity. In particular, the finding that $72.88 \%$ of all haplotypes were unique not only indicated high diversity within each ecotype population but also inferred that their genetic characteristics followed their geographical and ecological distributions.

However, no significant divergence in $F_{S T}$ was found among the 4 Naqu yak populations, and their genetic distances recapitulated the geographic distances between populations. This finding is consistent with the grazing features on the Tibetan Plateau, including the nomadic process, which results in gene exchange among domestic animal populations accompanied by human migration (China National Commission of Animal Genetic Resources, 2011).
In addition, according to the phylogenetic network constructed using the maximum likelihood method, two D-loop haplotype haplogroups were identified from the 59 Naqu yak haplotypes; this finding was consistent with previous studies of the two known domestic sites in the Chinese Tibetan yak (e.g., Song et al., 2014; Lai et al., 2005). Finally, no significant differences were found based on Tajima's D test $(P>0.10)$ of D-loop sequences in those populations, indicating that no historical population expansion occurred in the Naqu yak population.

\section{CONCLUSION}

The Naqu yak has multiple maternal origins and high diversity within different geographic ecotype yak populations. Additionally, the yaks are highly diverse within populations, although they are not heavily divergent, because all populations share the highest frequency haplotypes. Our work underlines the importance of Naqu yak genetic diversity studies not only to obtain a better understanding of the current domestic Naqu yak status but also to enhance and provide data for the development of biological genetic conservation strategies in the QinghaiTibet Plateau.

\section{ACKNOWLEDGMENTS}

This work was supported by National Technical System for Beef and Yak Industry (CARS-37), and the National Natural Science Foundation of China (No. 31172195).

Statement of conflict of interest

Authors have declared no conflict of interest.

\section{REFERENCES}

Almarzook, S., Reissmann, M. and Brockmann, G.A., 2017. Diversity of mitochondrial DNA in three Arabian horse strains. J. appl. Genet., 58: 273-276. https://doi.org/10.1007/s13353-016-0384-z

Bandelt, H.J., Forster, P. and Röhl, A., 1999. Medianjoining networks for inferring intraspecific phylogenies. Mol. Biol. Evol., 16: 37-48. https:// doi.org/10.1093/oxfordjournals.molbev.a026036

Cheng, S.R., Zeng, Y.F., Wang, X.R., Zhang, L.P. and $\mathrm{Wu}$, J.P., 2014. Genetic diversity and cluster analysis of six yak populations in Gansu province using mitochondrial D-loop sequence. Acta Agric. Boreali-Sin., 29: 16-21.

China National Commission of Animal Genetic Resources, 2011. Animal genetic resources in 
China: Sheep and goats. Chinese Agricultural Press, Beijing.

Deng, J., Feng, J., Li, L., Zhong, T., Wang, L., Guo, J., Ba, G., Song, T. and Zhang, H., 2017. Polymorphisms, differentiation, and phylogeny of 10 Tibetan goat populations inferred from mitochondrial D-loop sequences Mitochond. DNA A: DNA Mapp. Seq. Analysis, 30: 1-7.

Elsner, J., Hofreiter, M., Schibler, J. and Schlumbaum, A., 2017. Ancient mtDNA diversity reveals specific population development of wild horses in Switzerland after the Last Glacial Maximum. PLoS One, 12: e0177458. https://doi.org/10.1371/ journal.pone.0177458

Eusebi, P.G., Cortés, O., Dunner, S. and Cañón, J., 2017. Genetic diversity of the Mexican Lidia bovine breed and its divergence from the Spanish population. J. Anim. Breed. Genet., 134: 332-339. https://doi.org/10.1111/jbg.12251

Excoffier, L. and Lischer, H.E.L., 2010. A new series of programs to perform population genetics analyses under Linux and Windows. Mol. Ecol. Resour., 10: 564-567. https://doi.org/10.1111/j.17550998.2010.02847.x

Gao, Y.S., Jia, X.X., Tang, X.J., Fan, Y.F., Lu, J.X., Huang, S.H. and Tang, M.J., 2017. The genetic diversity of chicken breeds from Jiangxi, assessed with BCDO2 and the complete mitochondrial DNA D-loop region. PLoS One, 12: e0173192. https:// doi.org/10.1371/journal.pone.0173192

Guangxin, E., Na, R.S., Zhao, Y.J., Gao, H.J., An, T.W. and Huang, Y.F., 2016. Complete mitochondrial genome of the a rare subspecies of genus Bos, Tianzhu white yak from Tibetan area in China. Mitochond. DNA A: DNA Mapp. Seq. Analysis, 27: 1443-1444.

Guo, S., Savolainen, P., Su, J., Zhang, Q., Qi, D., Zhou, J., Zhong, Y., Zhao, X. and Liu, J., 2006. Origin of mitochondrial DNA diversity of domestic yaks. BMC Evol. Biol., 6: 73. https://doi. org/10.1186/1471-2148-6-73

Hai, T., Zhang, C.F., Xin, J.W., Ji, Q.M., Chai, Z.X., Zeng, X.B. and Zhong, J.C., 2014. Genetics diversity and phylogenetic analysis of mtDNA ND5 of Tibetan Yak. Southwest China J. agric. Sci., 27: 2666-2672.

Hernández, C.L., Dugoujon, J.M., Novelletto, A., Rodríguez, J.N., Cuesta, P. and Calderón, R., 2017. The distribution of mitochondrial DNA haplogroup $\mathrm{H}$ in southern Iberia indicates ancient human genetic exchanges along the western edge of the Mediterranean. BMC Genet., 18: 46. https://doi. org/10.1186/s12863-017-0514-6

Huang, X.D., Tan, H.Y., Long, R., Liang, J.B. and Wright, A.D., 2012. Comparison of methanogen diversity of yak (Bos grunniens) and cattle (Bos taurus) from the Qinghai-Tibetan plateau, China. BMC Microbiol., 12: 237. https://doi. org/10.1186/1471-2180-12-237

Jia, X.X., Lu, J.X., Tang, X.J., Fan, Y.F., Huang, S.H., Ge, Q.L. and Gao, Y.S., 2017. Genetic diversity of Jiangsu native chicken breeds assessed with the mitochondrial DNA D-loop region. Br. Poult. Sci., 13: $1-6$.

Khaire, D., Atkulwar, A., Farah, S. and Baig, M., 2017. Mitochondrial DNA analyses revealed low genetic diversity in the endangered Indian wild ass Equus hemionus khur. Mitochond. DNA A: DNA Mapp. Seq. Analysis, 28: 681-686.

Kim, J.H., Lee, S.S., Kim, S.C., Choi, S.B., Kim, S.H., Lee, C.W., Jung, K.S., Kim, E.S., Choi, Y.S., Kim, S.B., Kim, W.H. and Cho, C.Y., 2016. Haplogroup classification of Korean cattle breeds based on sequence variations of mtDNA control region. Asian-Australas. J. Anim. Sci., 29: 624-630. https:// doi.org/10.5713/ajas.16.0281

Lai, S.J., Chen, S.Y., Liu, Y.P. and Yao, Y.G., 2007. Mitochondrial DNA sequence diversity and origin of Chinese domestic yak. Anim. Genet., 38: 77-80. https://doi.org/10.1111/j.1365-2052.2007.01555.x

Lai, S.J., Wang, L., Liu, Y.P. and Li, X.W., 2005. Study on mitochondrial DNA genetic polymorphism of some yak breeds in China. Acta Genet. Sin., 32: 463-470.

Larkin, M.A., Blackshields, G., Brown, N.P., Chenna, R., McGettigan, P.A., McWilliam, H., Valentine, F., Wallace, I.M., Wilm, A., Lopez, R., Thompson, J.D., Gibson, T.J. and Higgins, D.G., 2007. Clustal $\mathrm{W}$ and Clustal $\mathrm{X}$ version 2.0. Bioinformatics, 23: 2947-2948. https://doi.org/10.1093/bioinformatics/ btm 404

Lyimo, C.M., Weigend, A., Msoffe, P.L., Eding, H., Simianer, H. and Weigend, S., 2014. Global diversity and genetic contributions of chicken populations from African, Asian and European regions. Anim. Genet., 45: 836-848. https://doi. org/10.1111/age. 12230

Ming, L., Yi, L., Sa, R., Wang, Z.X., Wang, Z. and Ji, R., 2017. Genetic diversity and phylogeographic structure of Bactrian camels shown by mitochondrial sequence variations. Anim. Genet., 48: 217-220. https://doi.org/10.1111/age.12511

Mipam, T.D., Wen, Y., Fu, C., Li, S., Zhao, H., Ai, Y., Li, L., Zhang, L. and Zou, D., 2012. Maternal 
phylogeny of a newly-found yak population in china. Int. J. mol. Sci., 13: 11455-11470. https:// doi.org/10.3390/ijms130911455

Nguluma, A.S., Msalya, G., Lyimo, C.M., Huang, Y., Zhao, Y., Chen, L., Guangxin, E. and Chenyambuga, S.W., 2017. Phylogenetic relationship and variation among four strains of small East African goats of Tanzania based on mitochondrial DNA. AsianAustralas. J. Anim. Sci., https://doi.org/10.5713/ ajas. 17.0327

Posada, D., 2008. jModelTest: Phylogentic model averaging. Mol. Biol. Evol., 25: 1253-1256. https:// doi.org/10.1093/molbev/msn083

Qian, J.X., Qian, H.P., Xu, Z.W. and Yuan, B.B., 2013. Phylogenetic relationship of Bos grunniens in Qinghai based on mitochondrial cox1 and nad1 Genes. China Anim. Husband. Vet. Med., 40: 151154.

Rozas, J. and Rozas, R., 1995. DnaSP, DNA sequence polymorphism: an interactive program for estimating Population Genetics parameters from DNA sequence data. Comput. Applic. Biosci., 11: 621-625.

Sambrook, J. and Russell, D., 2001. Molecular cloning: A laboratory manual, $3^{\text {rd }}$ ed. Cold Spring Harbor Laboratory Press, New York.

Schaan,A.P., Costa, L., Santos, D., Modesto, A., Amador, M., Lopes, C., Rabenhorst, S.H., Montenegro, R., Souza, B.D.A., Lopes, T., Yoshioka, F.K., Pinto, G., Silbiger, V. and Ribeiro-Dos-Santos, Â., 2017. mtDNA structure: the women who formed the Brazilian Northeast. BMC Evol. Biol., 17: 185. https://doi.org/10.1186/s12862-017-1027-7

Slatkin, M., 1995. A measure of population subdivision based on microsatellite allele frequencies. Genetics, 139: 457-462.

Song, Q.Q., Zhong, J.C., Zhang, C.F., Xin, J.W., Ji, Q.M. and Chai, Z.X., 2014. Analysis on genetic diversity and phyletic evolution of mitochondrial DNA from Tibetan yaks. Acta Theriol. Sin., 34: 356-365.

Tamura, K., Peterson, D., Peterson, N., Stecher, G.,
Nei, M. and Kumar, S., 2011. MEGA5: Molecular Evolutionary Genetics Analysis using Maximum Likelihood, Evolutionary Distance, and Maximum Parsimony Methods. Mol. Biol. Evolut., 28: 27312739. https://doi.org/10.1093/molbev/msr121

Tamura, K. and Nei, M., 1993. Estimation of the number of nucleotide substitutions in the control region of mitochondrial DNA in humans and chimpanzees. Mol. Biol. Evolut., 10: 512-526.

Tsai, T. and St John, J.C., 2016. The role of mitochondrial DNA copy number, variants, and haplotypes in farm animal developmental outcome. Domest. Anim. Endocrinol., 56(Suppl): S133-146. https:// doi.org/10.1016/j.domaniend.2016.03.005

Tu, S.Y., Wang, Q., Chai, Z.X. and Zhong, J.C., 2016. Analysis on genetic diversity and phyletic evolution of mitochondrial DNA D-loop region from Zhongdian Yak. China Herbivore Sci., 36: 5-10.

Wang, L.P., Geng, R.Q., Chang, H., Ji, D.J., Li, Y.H. and Chang, C.F, 2013. Analysis on genetic diversity and phylogenetic relationship of Xingjiang bazhou Yak based on mitochondrial DNA. Acta Ecol. Anim. Domast., 34: 17-20.

Xie, Y., Hoberg, E.P., Yang, Z., Urban, Jr, J.F. and Yang, G., 2017. Ancylostoma ailuropodaen sp. (Nematoda: Ancylostomatidae): A new hookworm parasite isolated from wild giant pandas in Southwest China. Parasit. Vectors, 10: 277. https:// doi.org/10.1186/s13071-017-2009-8

Zhang, C.F., Xu, L.J., Ji, Q.M., Xin, J.W. and Zhong, J.C., 2012. Genetic diversity and evolution relationship on mtDNA D-loop in Tibetan yaks. Acta Ecol. Sin., 32: 1387-1395. https://doi. org/10.5846/stxb201107131039

Zhang, J., Jiao, T. and Zhao, S., 2016. Genetic diversity in the mitochondrial DNA D-loop region of global swine (Sus scrofa) populations. Biochem. Biophys. Res. Commun., 473: 814-820. https://doi. org/10.1016/j.bbrc.2016.03.129 